\title{
Headache occurring during rehabilitation in a migrainous patient with cervical spinal cord injury
}

\author{
Ioana C Stănescu ${ }^{1,2}$, Angelo Bulboacăa ${ }^{1,2}$, Corina Ursu², Dana Fodor ${ }^{1}$
}

Corresponding author: Ioana Stanescu, E-mail: ioana.stanescu.umfcluj@gmail.com

\section{1 "Iuliu Hatieganu" University of Medicine and Pharmacy, Cluj-Napoca, Romania \\ ${ }^{2}$ Clinical Rehabilitation Hospital, Cluj-Napoca, Romania}

\begin{abstract}
Traumatic vertebral artery (VA) dissection is a severe consequence of a cervical injury, which usually involves the vertebrae and spinal cord. Traumatic VA dissection has been frequently underdiagnosed or misdiagnosed, mainly because many patients remain asymptomatic. The consequence of VA dissection is ischemia in the territory supplied by the affected artery. The VA dissection presents most often as a vertebrobasilary transient ischemic attack or ischemic stroke, usually preceded by local symptoms such as neck pain or headache. Headache is a common symptom in patients with cervical artery dissection, but its characters are not specific. Diagnosis of VA dissection usually requires a CT-angiography. Delayed onset of symptoms with a variable asymptomatic interval, ranging from several days to 3 months, has been reported. The risk of stroke remains high, especially in the first weeks after the confirmation of VA dissection. Treatment of traumatic VA dissections include anticoagulation or antiplatelet therapy and revascularization techniques. We present the case of a patient with spinal cord injury and operated C5 fracture, who was diagnosed with unilateral VA dissection 6 months after the traumatic event, during the rehabilitation program.
\end{abstract}

Key words: vertebral artery dissection, cervical spinal cord injury, headache, rehabilitation,

\section{Introduction}

Traumatic vertebral artery (VA) dissection is a relatively rare sequela of cervical trauma and can occur as a result of different trauma types that cause excessive cervical rotation or extreme flexionextension of the neck. Traumatic VA dissection has been frequently underdiagnosed or misdiagnosed, mainly because many patients remain asymptomatic if the VA is damaged only unilaterally (1).

Headache is the common symptom in patients with cervical artery dissection (2)._Delayed onset of symptoms with a variable asymptomatic interval, ranging from several days to 3 months, has been reported (1). We present the case of a patient who showed delayed symptoms of VA dissection, 6 months after the traumatic event.

\section{Case report}

A 37-year-old male patient with a history of migraine was involved in a car accident with traumatic cervical spinal cord injury, with fracture of the C5 vertebra. Neurosurgical intervention was performed in emergency with decompression and stabilization of the spine by posterior fixation with rods and screws (Figure 1).

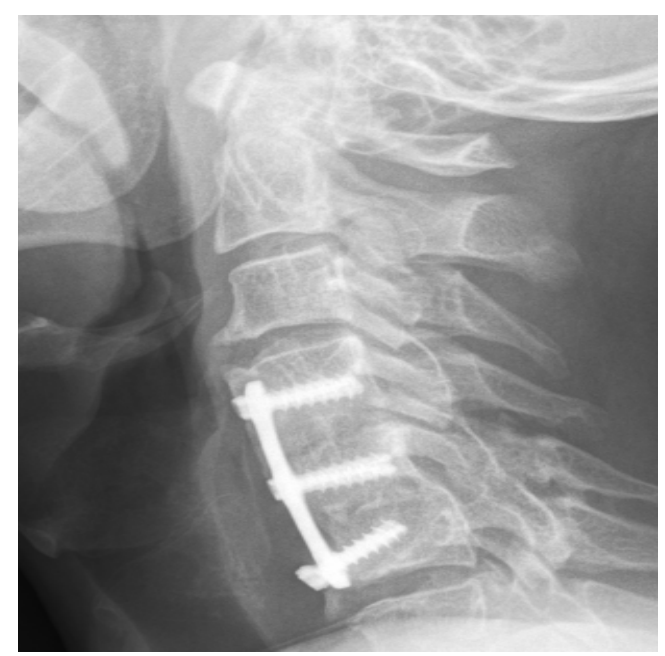

Fig. 1: Radiographies of the cervical spinal cord showing C5 fracture and stabilization of the spine with rods and screws

After surgery, the patient had an American Spinal Injury Association (ASIA) grade B. Six months after his traumatic event, the patient improved to an ASIA grade C, was admitted to our Rehabilitation Hospital, and started a physical therapy program. His neurological examination showed important motor deficit in the distal upper limbs (especially for wrist 
extensors and finger flexors), spastic motor deficit in the lower limbs, brisk reflexes, bilateral Babinski sign, decreased pain and temperature sensations below D4 level, with preserved light touch and proprioception in the lower limbs, sphincterian troubles with urge micturition. He was able to walk a few steps with a rollator. Electrocardiogram (ECG) showed asymptomatic sinus bradycardia of $42 / \mathrm{min}$. At admission, the patient's complaints were mild occipital headache and right side cervical pain. No signs of cranial nerve involvement were observed. The patient also reported an increased frequency of migraine attacks after the traumatic event.

The physical therapy program consisted of sedative massage of the cervical-dorsal-lumbar spine, passive postural changes and mobilization of the upper and lower limbs to prevent the effects of prolonged immobilization, respiratory gymnastics to maintain the respiratory function. For recovery of sphincter disorders, pelvic floor toning exercises and interferential electrostimulation were performed. Also, the patient was included in a virtual therapy and occupational therapy program to increase mobility and recover balance. For recovery of walking, the parallel bar system was used, as well as a robotic system which allowed support of the lower limb joints, particularly the knees, and intelligent control of walking assistance depending on the patient's movement capacity. The rehabilitation program was conducted over three weeks; at discharge, the indication was to continue the kinesitherapy program at home.

No manipulative techniques for the cervical area were performed.

Three days after admission, the patient complained of worsening of headache and right cervicalgia; the headache had different features than his usual migraine attack (increased intensity, continuous type, no pulsating character). No changes in neurological exam were noted. The pupils were symmetrical and reactive. Cervical spine plain radiographs were identical to previous examinations. Native cerebral CT scan was normal, showing no hydrocephalus.

Cervical MRI was performed, evidencing T2 hyperintensities at C5-C6 level of the spinal cord, consistent with traumatic myelopathy; there was no syringomyelia (Figure 2).

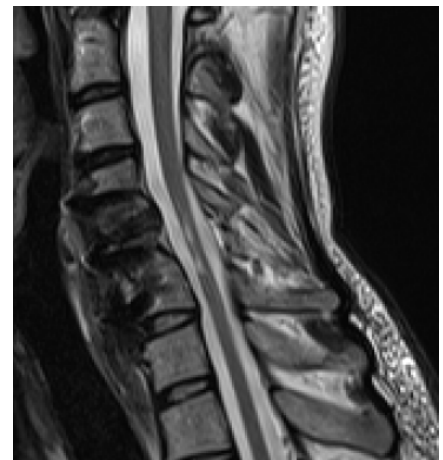

Fig. 2: Cervical MRI, sagital sequences, showing T2 hyperintensities in the spinal cord, correspponding to posttraumatic myelopathy

On axial sequences, absence of flow in the proximal V2 segment of the right vertebral artery was observed, and in distal V2 the blood flow was filiform. The left VA was dominant, without flow abnormalities (Figure 3).

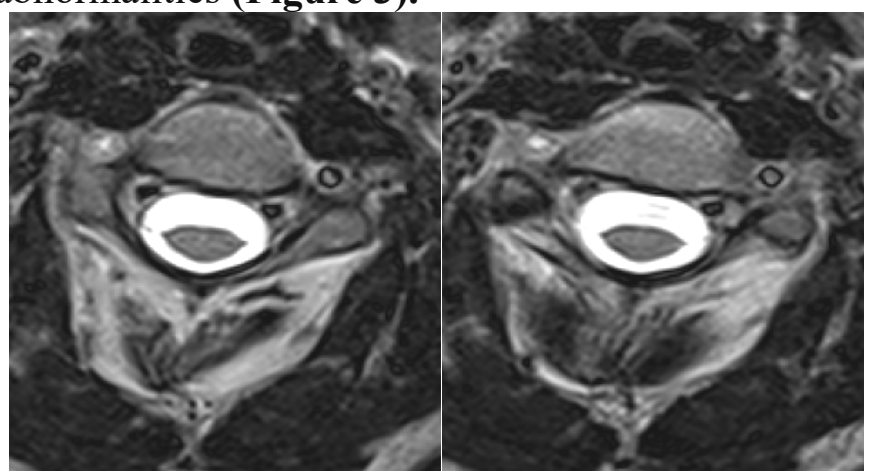

Fig. 3: Cervical MRI, axial sequences at C5-C6 level, showing normal flow in the left VA and absence of flow in the right VA, corresponding to VA occlusion

We obtained previous MRI of the patient, performed one month after surgical intervention (Figure 4), and we compared the aspect of vertebral arteries on axial sections, at the same level; we have not noticed any flow abnormalities, and we have presumed that, at the time when this MRI has been performed, the right VA flow have been normal.

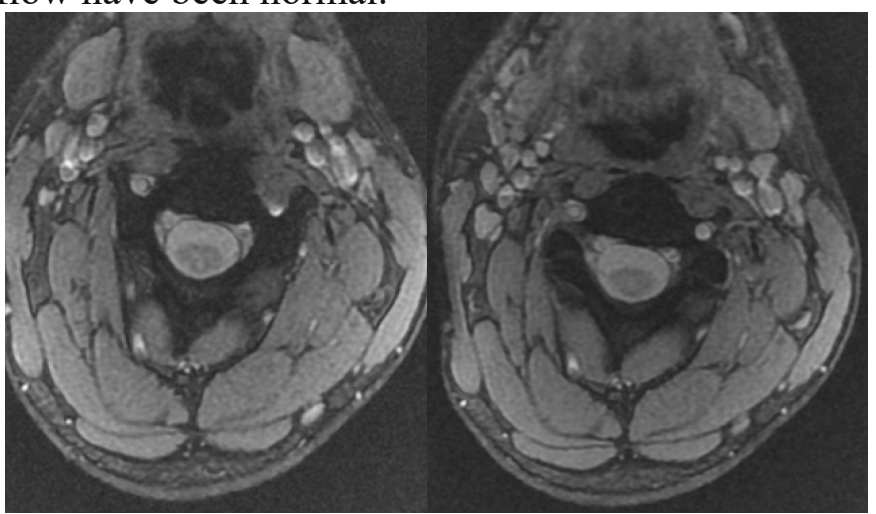

Fig . 4: Cervical MRI performed 1 month after traumatism, axial sequences at C5-C6 level, showing presence of blod flow in V2 segment of right VA 
Doppler ultrasound examination of cervical and cerebral arteries revealed the absence of flow in the V2 segment of the right VA, normal flow in the dominant left VA, and increased intima-media thickness (IMT) bilaterally. Normal flow in both V3 segments and basilar artery was observed on transcranial Doppler ultrasound (TCD).

An angio-CT scan of cervical vessels was then performed, and dissection of the V2 segment of the non-dominant right VA was confirmed (Figure 5).
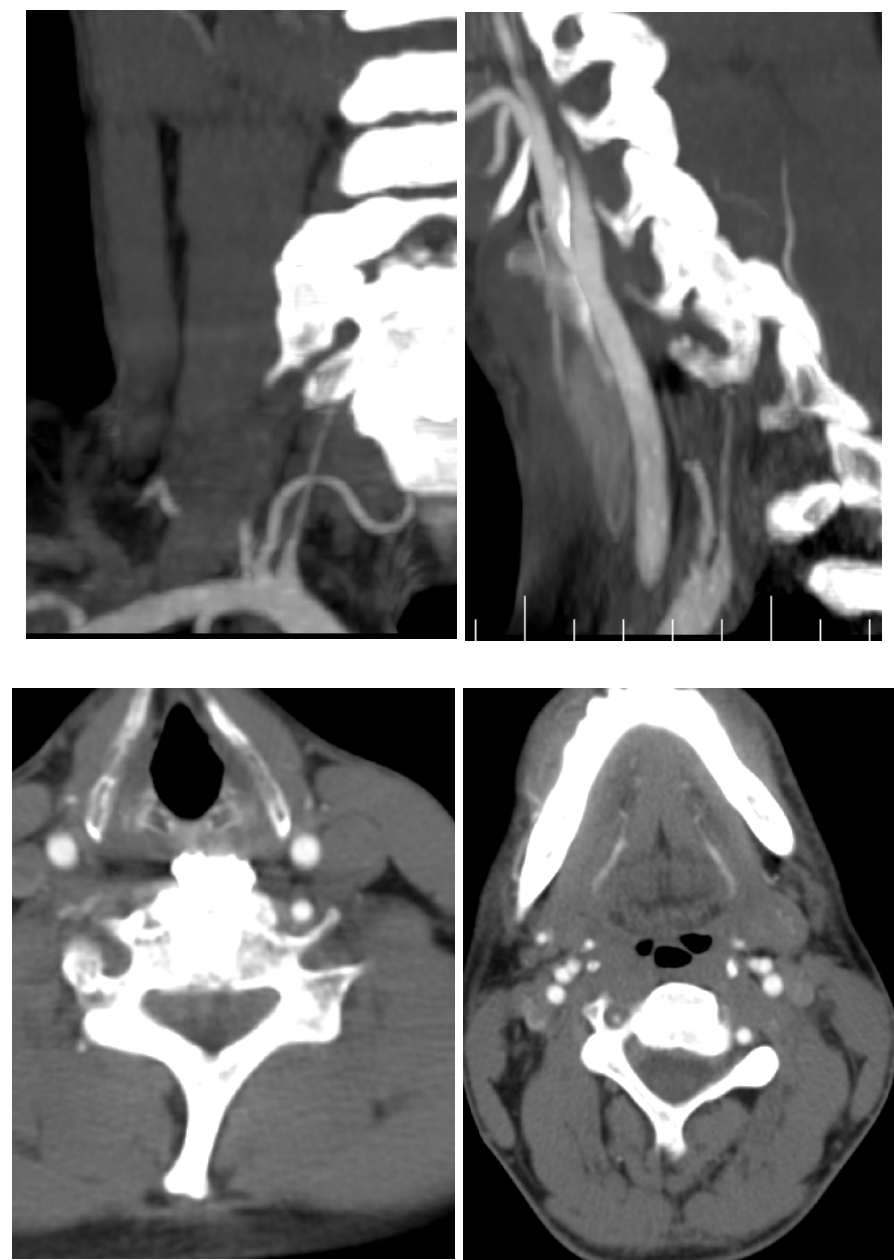

Fig. 5. Angio-CT scan of the neck showing signs of right VA dissection in $\mathrm{V} 2$ segment ( $\mathrm{A}$ - coronal view, $\mathrm{B}$ - lateral view, C, D - axial view)

Antiplatelet treatment for VA dissection was started in our patient, associated with symptomatic analgesic treatment, with gradual improvement in the patient's headache. The patient was discharged after 21 days, with improved neurological status, but with the same ASIA grade C. Prophylactic treatment with curcumin for increased frequency of migraine attacks was initiated.
The patient returned for follow-up at 3 months. No new neurological symptoms were present from discharge, and his neurological condition remained stable. Doppler ultrasound of cervical arteries showed no recanalization of the V2 segment of the injured right VA. Antiplatelet treatment was stopped. The patient in stable condition was then referred for balneotherapy in a spa resort $(3,4)$ in the form of balneation and crenotherapy, with specific natural factors.

\section{Results and discussion}

Arterial injuries are not very rare consequences of cranial or spinal cord injuries. Cervical arteries are also exposed to traumatic factors, especially the extracranial parts of the VA which passes through the transverse foramina of the cervical vertebrae, having a higher incidence of dissection, especially in the V2 segment. Fractures involving the transverse foramina or the lateral vertebral masses, or vertebral subluxations may damage/tear/traction the artery, causing dissection (5). Some VA dissections may extend to more than one segment (6). During arterial dissection, a tear forms in the arterial wall, leading to intrusion of blood within the layers of the arterial wall resulting in an intramural hematoma (7).

The consequence of VA dissection is ischemia in the territory supplied by the affected artery: vertebrobasilary stroke or transient ischemic attack (TIA). The mechanism of stroke is presumed to be embolic from a local thrombus or hemodynamic by a decrease in blood flow in the absence of collateral circulation.

Clinically, VA dissection most often produces a vertebrobasilary TIA or ischemic stroke, usually preceded by local symptoms such as neck pain or headache. Headache is the common symptom in patients with cervical artery dissection (CAD), but the characteristics of pain associated with CAD are not specific and can sometimes resemble migraine or even cluster headache (2). A recent study showed that more specific headache characteristics in patients with intracranial vertebral artery dissection included: headache in the occipitonuchal region, with unilateral, pulsatile and acute onset, with severe intensity, and without nausea or vomiting (8).

We assumed that patient's cervicalgia and headache were related to right VA dissection although the patient was a migraineur. 
The persistence and intensity of pain raised the suspicion of another etiology than a migraine attack. First we looked for a possible dislocation of the fixation material used to stabilize the cervical spine, but the radiographs indicated a normal position of the rods and screws. A new cervical MRI was performed to rule out a possible post-traumatic syrinx, but the spinal cord showed only chronic post-traumatic changes.

However, headache rarely occurs in isolation, without focal neurological signs (2). If the damage is unilateral and has induced occlusion of the nondominant VA, it may not cause ischemic symptoms. Outcome in VA dissections is dependent on perfusion of the contralateral VA: if inadequate, it can induce vertebrobasilary (VB) ischemia; dominant VA occlusion can result in a more severe clinical picture (5). In our case, Doppler ultrasound and angio-CT scan showed that the left VA was dominant, and the basilar artery had normal perfusion, so this could explain why our patient did not have ischemic symptoms.

The particularity of this case was the long time interval between the traumatism and VA dissection. A latent period between the time of injury and the appearance of clinical manifestations of a few hours or days is typically seen (9). Delayed onset of symptoms with a variable lucid interval, ranging from several days to 3 months, has also been reported (2). In a series of 500 patients with whiplash injury acquired in car accidents, the incidence of cervical arterial dissection occurring within 12 months after the traumatism was $1.6 \%$, and the mean delay between the trauma and arterial dissection diagnosis was $72 \pm 99$ days (10). In $87.5 \%$ of cases from this study, the dissection was complicated by brain infarction, and the risk of cerebrovascular events was still increased 4-12 months after injury (10).

The risk of stroke remained important in our patient, especially in the first weeks after the confirmation of VA dissection. In a study of over 800 patients with traumatic cervical artery dissection without stroke, the risk of stroke was $1.43 \%$, not significantly different from that of non-traumatic dissections (11), and was maximal during the first 2 weeks. In CADISS (Cervical Artery Dissection in Stroke Study), which randomized patients presenting with cervical artery dissection, an overall stroke recurrence rate of $2 \%$ at 3 months was observed, with all recurrences occurring within the first 10 days (12).
Therapeutic strategies in traumatic VA dissections include anticoagulation or antiplatelet therapy, revascularization techniques (stent-assisted arterial reconstruction), and endovascular permanent arterial occlusion (6). According to the literature, recanalization may occur weeks and even months (max. 24 months) after arterial dissection (10).

Migraine attacks were also disabling for the patient, with increased frequency after the traumatism. Prophylactic treatment had to be considered in this case. Our patient presented with bradycardia, so the use of beta-blockers for migraine prophylaxis was inappropriate. According to our recent research, curcumin was shown to be effective in migraine prophylaxis (13) and in migraine attacks due to its important anti-inflammatory effects (14), being also well tolerated by patients.

Patients with spinal cord injury need long recovery periods. Functional risk complications are possible, which cause long term or permanent disability requiring specialized medical rehabilitation care. Rehabilitation treatment in SPA resorts has also proved to be effective in ameliorating disability in patients with important neurological deficits (3). Also, SPA treatment is effective in different types of migraine, while behavioral interventions will be beneficial in reducing the risk of cronicisation (15).

\section{Conclusions}

Vertebral artery dissection are a severe complication of traumatisms in the cervical area. Diagnosing a VA injury could be difficult, because some dissections are asymptomatic, others have minor symptoms (as headache or cervicalgia), or in other cases the severity of the neurological picture in SCI could mask vascular symptoms. Because VA dissections carry an important risk of stroke in the vertebrobasilary system, screening for VA injury after cervical spine fractures should be performed when necessary, on a case-by-case basis (16). Screening criteria have been recently published, and CT-angiography is recommended for the detection of a VA dissection (17). An important point is the free interval between the traumatic event and the occurrence of symptoms of VA dissection, which was particularly long in our patient. At least 3 months of treatment is safe to prevent ischemic events in affected patients, but the choice between antiplatelet versus anticoagulant therapy is under debate. VA dissections carried good prognosis in treated patients. 


\section{Declaration of conflict of interests}

There is no conflict of interest for any of the authors regarding this paper.

\section{Informed consent}

An informed consent was obtained from the patient included in this study.

\section{References}

1. Sadaharu Tabuchi, Hiroyuki Nakayasu. Traumatic vertebral artery dissection and cerebral infarction following head and neck injury with a lucid interval. Acute Med Surg. 2015 Apr; 2(2): 127-130

2. Jatuzis D, Valaikiene J. Migraine-like presentation of vertebral artery dissection after cervical manipulative therapy. Perspectives in Medicine. 2012; 1 (1-12): 452-454

3. Dogaru G, Ispas A, Bulboacă A, Motricală M, Stănescu, I. Influence of balnear therapy at Băile Tusnad on quality of life of post-stroke patients. Balneo Research Journal. 2017;8 (4):201-205.

4. Dogaru G, Motricală M, Molnár Á, Rus V. Effects of mineral water from spring 3 in Băile Tuşnad on experimentally induced alcoholic liver disease. Balneo Research Journal. 2017;8(3):125-128

5. Lammy S, Bhatt P. Delayed presentation of vertebral artery dissection. Journal of Neurological Surgery. Part A: Central European Neurosurgery. 2012; 4(2):955-957

6. Cohen JE, Fraifeld S, Itshayek E. Endovascular Management of Traumatic Vertebral Artery Dissections. Treatment options in cases when anticoagulation is not a good solution and the risk of stroke is high. Endovascular Today. 2014,Sept; 68-74.

7. Kwan-Woong Park, Jong-Sun Park, Sun-Chul Hwang, Soo-Bin Im, Won-Han Shin, Bum-Tae Kim. Vertebral Artery Dissection: Natural History, Clinical Features and Therapeutic Considerations. J Korean Neurosurg Soc. 2008 Sep; 44(3): 109-115

8. Matsumoto, H., Hanayama, H., Sakurai, Y., Minami, H., Masuda, A., Tominaga, S., Hirata, Y. Investigation of the characteristics of headache due to unruptured intracranial vertebral artery dissection. Cephalalgia. 2018 Aug 6:333102418791818

9. Stanescu I, Bulboaca A, Kallo R, Dogaru G. Neurorehabilitation in stroke produced by vertebral artery dissection: case presentation. Balneo Research Journal. 2018; 9 (1), 34-37

10. Hauser V, Zangger P, Winter Y, Oertel W, Kesselring J. Late sequelae of whiplash injury with dissection of cervical arteries. Eur. Neurol. 2010; 64: 214-218.

11. Morris MA, Merkler AE, Gialdini G, Kamel H. Timing of Incident Stroke Risk After Cervical Artery Dissection Presenting Without Ischemia. Stroke. 2017;48 (3):551-555

12. Kennedy F, Lanfranconi S, Hicks C, Reid J, Gompertz P, Price C, et al. CADISS Investigators. Antiplatelets vs anticoagulation for dissection: CADISS nonrandomized arm and meta-analysis. Neurology. 2012;79 (7):686-689

13. Bulboacă AE, Bolboacă SD, Stănescu IC, Sfrângeu CA, Bulboacă AC. Preemptive Analgesic and Antioxidative Effect of Curcumin for Experimental Migraine. Biomed Res Int. 2017;2017:4754701

14. Bulboacă, A. E., Bolboacă, S. D., Stănescu, I. C., Sfrângeu, C. A., Porfire, A., Tefas, L., \& Bulboacă, A. C. (2018). The effect of intravenous administration of liposomal curcumin in addition to sumatriptan treatment in an experimental migraine model in rats. International journal of nanomedicine. 2018; 13, 3093-3103

15. Stanescu I, Dogaru G. Treatment in chronic migraine choice of rehabilitation strategies. Balneo Research Journal. 2015; 6 (4), 217-223.

16. Lockwood MM, Smith GA, Tanenbaum J, Lubelski D, Seicean A, Pace J, Benzel EC, Mroz TE, Steinmetz MP. Screening via CT angiogram after traumatic cervical spine fractures: narrowing imaging to improve cost effectiveness. Experience of a Level I trauma center. J Neurosurg Spine. 2016 Mar:24(3):4905.

17. Brommeland T, Helseth E, Aarhus M, Moen KG, Dyrskog S, Bergholt B, Olivecrona $\mathrm{Z}$, Jeppesen E. Best practice guidelines for blunt cerebrovascular injury (BCVI). Scand J Trauma Resusc Emerg Med. 2018 Oct 29;26(1):90 